\title{
Analyse pratique de pieux chargés latéralement dans un milieu pulvérulent
}

\author{
A. Alem - M. Gherbi \\ Equipe de génie civil, Laboratoire de mécanique, Le Havre
}

\begin{abstract}
Résumé
Cette communication présente un exemple de solutions analytiques développées pour l'analyse de pieux chargés latéralement. Les solutions sont basées sur une modélisation simplifiée de l'interaction sol-structure. Une bonne concordance entre les résultats théoriques et les données d'essais pris dans la littérature atteste de la validité des solutions proposées. Les principaux résultats, permettant un calcul rapide de tels ouvrages, sont donnés sous forme d'abaques ou de tables.
\end{abstract}

\begin{abstract}
Some results of a simplified analysis method of a single laterally loaded piles embeded in cohesionless soil are presented. The pile is assumed as a beam on elastoplastic foundation. Good agreement between the theoretical solutions and data from published case histories attests to the validity of the method. The results are presented in the form of nondimensional charts that permit hand calculations and rapid verification of structural design of piles.
\end{abstract}

\section{Introduction}

Depuis des décennies, diverses méthodes ont été développées pour l'analyse du comportement de pieux chargés latéralement. Ces méthodes peuvent être classées en trois groupes:

- les méthodes classiques qui supposent le sol entièrement à l'état limite autour du pieu (concept de pression uitime sur toute la hauteur du pieu),

- les méthodes basées sur la théorie de l'élasticité qui restent peu utilisées,

- les méthodes aux modules de réaction qui sont les plus répandues. Ces méthodes assimilent le pieu à une poutre sur appuis élastiques linéaires ou non. Elles permettent de faire des calculs eñ déplacement, ce qui est un point capital pour le dimensionnement de tels pieux. Elles nécessitent par contre une détermination préalable des courbes de réactions qui lient, à chaque niveau, le déplacement horizontal du pieu $(\mathrm{Y})$ à la réaction exercée par le sol $(\mathrm{P})$. Cet article expose quelques résultats d'une méthode au module de réaction simple de calcul de pieux chargés latéralement. Les courbes P-Y employées sont très simples et les solutions sont analytiques. Les principaux résultats sont présentés sous forme d'abaques ou tabulés et sont d'un usage aisé. 


\section{Domaine d'application}

La solution analytique décrite ci-après repose sur les hypothèses suivantes:

- le pieu est considéré comme infiniment long, $L \geq 3 l_{0}$. L étant la fiche du pieu, $l_{0}=(4 \mathrm{EI} / \mathrm{Es})^{0.25}$ la longueur de transfert du pieu, $\mathrm{B}_{\mathrm{p}}$ le diamètre, EI la rigidité à la flexion du pieu et Es le module de réaction du sol en $\mathrm{N} / \mathrm{m}^{2}$.

- le sol est homogène à module de réaction constant. La méthode est ensuite étendue au cas d'un module de réaction croissant avec la profondeur comme il est généralement admis pour les sols pulvérulents.

- la pression limite varie linéairement avec la profondeur, $\mathrm{Pu}(z)=\alpha z$.

- les courbes de réaction sont définies par des courbes élastoplastiques pures (figure 1). Les paramètres, Es et $\mathrm{Pu}$, permettant de construire les courbes $\mathrm{P}-\mathrm{Y}$ à chaque niveau peuvent êtres déduit d'essais en place (le pressiomètre ou le pénétromètre) ou d'essais de laboratoire.

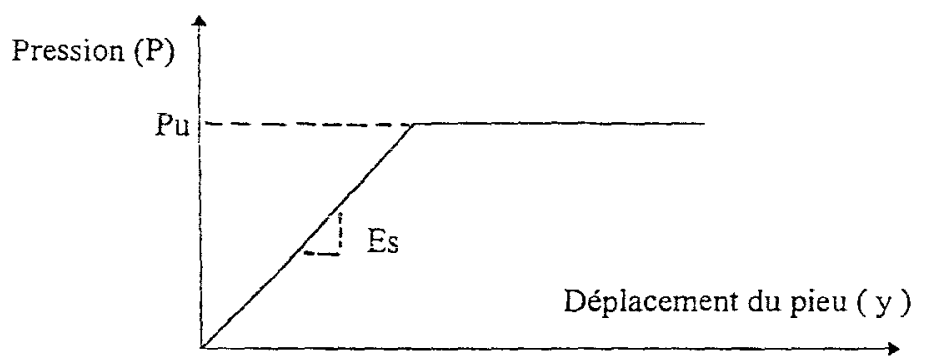

Figure 1 - Courbe Pression-déplacement

\section{Formulation et mise en équations}

Les expériences réalisées sur des pieux en acier, entre autre dans le cas de ducs d'albe de défense, ont révélé d'importants déplacements horizontaux des pieux au niveau et juste en dessous de la surface du sol. Ces déplacements entraînent des déformations plastiques et même des fissurations de la masse du sol environnante. Sur la base de ces constatations, nous définissons deux zones de pression différentes dans le sol entourant un pieu soumis à des charges latérales (figure 2 ).



Figure 2 : Distribution de la réaction du sol le long d'un pieu chargé latéralement 
Dans la zone 1 "sol est en état plastique", la pression du sol à la profondeur z est indépendante du déplacement latéral du pieu et est égale à la pression limite à cette profondeur. Pour un milieu pulvérulent, la pression limite s'écrit :

$\operatorname{Pu}(z)=\alpha z$

$\alpha$ peut être déduit d'essais en place, sinon il peut être estimé par l'expression suivante :

$\alpha=\mathrm{K}_{\mathrm{p}}^{2} \gamma^{\prime} \mathrm{B}_{\mathrm{p}}$

avec $\mathrm{K}_{\mathrm{p}}=\tan ^{2}(45+\phi / 2), \gamma^{\prime}$ le poids volumique déjaugé du sol et $\phi$ l'angle de frottement interne du sol.

L'équation d'équilibre des pressions sur le pieu s'écrit sous la forme :

$\mathrm{EI} \frac{d^{4} y_{1}}{d z^{4}}=-\mathrm{Pu}(\mathrm{z})=-\alpha z$

On obtient par intégrations successives :

$\mathrm{EI} \frac{d^{3} y_{1}}{d z^{3}}=-\alpha \frac{z^{2}}{2}+\mathrm{C}_{1}$

$\mathrm{EI} \frac{d^{2} y_{1}}{d z^{2}}=-\alpha \frac{z^{3}}{6}+\mathrm{C}_{1} z+\mathrm{C}_{2}$

$\mathrm{EI} \frac{d y_{1}}{d z}=-\alpha \frac{z^{4}}{24}+\mathrm{C}_{1} \frac{z^{2}}{2}+\mathrm{C}_{2} z+\mathrm{C}_{3}$

EI $y_{1}(\mathrm{z})=-\alpha \frac{z^{5}}{120}+\mathrm{C}_{1} \frac{z^{3}}{6}+\mathrm{C}_{2} \frac{z^{2}}{2}+\mathrm{C}_{3} z+\mathrm{C}_{4}$

$y_{1}(z)$ est le déplacement du pieu à la profondeur $z$ dans la zone 1.

Dans la zone 2 "zone élastique", la pression du sol est proportionnelle au déplacement latéral du pieu.

L'équation d'équilibre des pressions sur le pieu s'écrit sous la forme :

EI $\frac{d^{4} y_{2}}{d z^{4}}=-$ Es $y_{2}(z)$

$y_{2}(z)$ est le déplacement latéral du pieu à la profondeur $z$ dans la zone 2 .

Dans le cas d'un pieu long, cette équation a pour solution :

$$
\begin{aligned}
\text { EI } y_{2}(\mathrm{z}) & =\mathrm{EI}^{-\beta \cdot z}\left[\mathrm{C}_{3} \sin (\beta \mathrm{z})+\mathrm{C}_{6} \cos (\beta \mathrm{z})\right] \\
\text { où } \beta & =\frac{1}{l_{0}}
\end{aligned}
$$

On obtient par dérivations successives les expressions des rotations, des efforts internes $V(z)$ et $M(z)$ et de la réaction du sol $P(z)$. 
En écrivant les conditions en tête, les conditions d'équilibre et de continuité du déplacement et de rotation du pieu à la profondeur $z_{p}$, il s'ensuit un système d'équations qui permet d'obtenir les constantes $C_{1}, C_{2}, C_{3}, C_{4}, C_{5}, C_{6}$ et $z_{p}$. Le comportement du pieu se trouve alors complètement défini.

Pour un pieu libre en tête et soumis à un effort et à un moment (figure 1), cas d'un pieu de duc d'albe, on trouve:

$\mathrm{C}_{1}=\mathrm{H}_{0} ; \mathrm{C}_{2}=\mathrm{M}_{0}$

$C_{3}=E I\left[-\beta A\left(z_{p}\right)-\beta B\left(z_{p}\right)\right]+\alpha \frac{z_{p}{ }^{4}}{24}-H_{0} \frac{z_{p}{ }^{2}}{2}-M_{0} z_{p}$

$C_{4}=E I\left[\left(1+\beta z_{p}\right) A\left(z_{p}\right)+\beta z_{p} B\left(z_{p}\right)\right]-\alpha \frac{z_{p}{ }^{5}}{30}+H_{0} \frac{z_{p}{ }^{3}}{3}+M_{0} \frac{z_{p}{ }^{2}}{2}$

$C_{s}=e^{-\beta \cdot z_{p}}\left[A\left(Z_{p}\right) \sin \left(\beta z_{p}\right)-B\left(Z_{p}\right) \cos \left(\beta z_{p}\right)\right]$

$C_{6}=e^{-\beta \cdot z_{p}}\left[A\left(z_{p}\right) \cos \left(\beta z_{p}\right)+B\left(z_{p}\right) \sin \left(\beta z_{p}\right)\right]$

dans lesquels :

$A\left(z_{p}\right)=\frac{\alpha z_{p}}{E S} ; \quad B\left(z_{p}\right)=\frac{1}{2 \beta^{2} E I}\left(-\alpha \frac{z_{p}^{3}}{6}+H_{0} z_{p}+M_{0}\right)$

$z_{p}$ est la racine positive de l'équation du $3^{\text {ème }}$ degré suivante :

$\beta \alpha \frac{z_{p}^{3}}{6}+\alpha \frac{z_{p}^{2}}{2}+\left(\frac{\alpha}{2 \beta}-\beta H_{0}\right) z_{p}-\left(\beta M_{0}+H_{0}\right)=0$

Cette équation admet les solutions suivantes :

pour $\mathrm{H}_{0}$ et $\mathrm{M}_{\mathrm{o}}$ vérifiant l'inégalité : $\quad \frac{2 \mathrm{H}_{0}}{\alpha} \geq\left[\frac{1}{2 \beta^{3}}+\frac{3 \mathrm{M}_{0}}{\alpha}\right]^{2 / 3}$

$\mathrm{z}_{\mathrm{p}}=2 \sqrt{\frac{2 \mathrm{H}_{0}}{\alpha} \cos \frac{\theta}{3}}-\frac{1}{\beta} \quad$ avec $\quad: \theta=\operatorname{Arccos}\left[\frac{\frac{1}{2 \beta^{3}}+\frac{3 \mathrm{M}_{0}}{\alpha}}{\left[\frac{2 \mathrm{H}_{0}}{\alpha}\right]^{3 / 2}}\right]$

et pour $\mathrm{H}_{0}$ et $\mathrm{M}_{0}$ vérifiant l'inégalité : $\frac{2 \mathrm{H}_{0}}{\alpha}<\left[\frac{1}{2 \beta^{3}}+\frac{3 \mathrm{M}_{0}}{\alpha}\right]^{2 / 3}$

$\mathrm{z}_{p}=\left[\frac{1}{2 \beta^{3}}+\frac{3 \mathrm{M}_{0}}{\alpha}+\left(\left(-\frac{2 \mathrm{H}_{0}}{\alpha}\right)^{3}+\left(\frac{1}{2 \beta^{3}}+\frac{3 \mathrm{M}_{0}}{\alpha}\right)^{2}\right)^{1 / 2}\right]^{1 / 3}+$
$\left[\frac{1}{2 \beta^{3}}+\frac{3 \mathrm{M}_{0}}{\alpha}-\left(\left(-\frac{2 \mathrm{H}_{0}}{\alpha}\right)^{3}+\left(\frac{1}{2 \beta^{3}}+\frac{3 \mathrm{M}_{0}}{\alpha}\right)^{2}\right)^{1 / 2}\right]^{1 / 3}-\frac{1}{\beta}$ 


\section{Cas d'un sol à module de réaction variant linéairement avec la profondeur ou d'un multicouche}

\section{4.l. Sol à module de réaction variant linéairement avec la profondeur}

Dans ce cas, l'équation (9) n'admet pas de solution analytique. Une solution approchée basée sur les résultats décrits précédemment a été obtenue. Le module de réaction variant linéairement avec la profondeur est remplacé par un module de réaction, équivalent, constant qui donne les mêmes déplacements en tête du pieu en considérant le sol élastique. Les expressions littérales du déplacement en tête du pieu (figure 1), s'écrivent comme suit :

- pour un sol à module de réaction constant :

$\mathrm{y}_{\mathrm{o}}=\frac{2 \mathrm{H}_{\mathrm{o}} \beta\left(\mathrm{h}_{\mathrm{o}} \beta+1\right)}{\mathrm{Es}}$

- pour un sol à module de réaction variant linéairement avec la profondeur :

$$
\begin{gathered}
\mathrm{y}_{\mathrm{o}}=\frac{2.4 \mathrm{H}_{0}}{(\eta)^{3 / 5}(\mathrm{EI})^{2 / 5}}+\frac{1.60 \mathrm{H}_{0} h_{0}}{(\eta)^{2 / 5}(\mathrm{EI})^{3 / 5}} \\
\text { avec } \mathrm{h}_{0}=\mathrm{M}_{0} / \mathrm{H}_{0}
\end{gathered}
$$

En imposant l'égalité des déplacements en tête du pieu, on déduit une relation implicite qui permet d'obtenir le module de réaction constant équivalent.

$$
h_{0}\left(\frac{\mathrm{Es}}{4 \mathrm{EI}}\right)^{0.5}+\left(\frac{\mathrm{ES}}{4 \mathrm{EI}}\right)^{0.25}=\mathrm{Es}\left(\frac{1.2}{(\eta)^{3 / 5}(\mathrm{EI})^{2 / 5}}+\frac{0.80 \mathrm{ho}_{\mathrm{o}}}{(\eta)^{2 / 5}(\mathrm{EI})^{3 / 5}}\right)
$$

\section{2. sol multicouche}

Pour un sol multicouches, il convient de prendre une valeur moyenne de module réaction du sol.

$$
E s_{m}=\frac{\sum E s_{i} \cdot \ell_{i}}{\sum \ell_{i}}
$$

où $E s_{i}$ est le module de réaction dañs la couche i et $\ell_{i}$ la hauteur de la couche $\mathrm{i}$.

\section{Exemple de calcul}

La méthode a été validée par comparaison des résultats de calculs avec des résultats d'essais pris dans la littérature (essais en vraies grandeurs et essais en centrifugeuses ). On présente ici, à titre d'exemple, une comparaison effectuée sur un essai en vraie grandeur. L'essai consiste en un chargement latéral d'un pieu fiché dans du sable, (Cox 1974). Le pieu a un diamètre de $610 \mathrm{~mm}$ et une rigidité à la flexion del $172 \mathrm{MNm}^{2}$. La fiche est de $21 \mathrm{~m}$ et les chargements sont appliqués à $0,305 \mathrm{~m}$ au-dessus de la surface du sol. Les paramètres de sol utilisés sont: $\gamma^{\prime}=10.5 \mathrm{kN} / \mathrm{m}^{3}, \varphi=39^{\circ}, \eta=26 \mathrm{MN} / \mathrm{m}^{2}$, Reese (1974) et Budhu (1987). Le coefficient de variation de la pression limite $\alpha$ est calculé par l'expression (2). 
Les figures 3, 4 et 5 donnent les résultats de calcul par la méthode proposée, les résultats expérimentaux ainsi que les calculs par la méthode P-Y (Reese 1974). Comme on peut le voir sur ces figures, les résultats de calcul par la méthode proposée sont en très bon accord avec les résultats d'essais.

D'une manière générale, une bonne concordance entre les résultats théoriques et les données d'essais a été observée.

Figure 3

Force en tête eñ fonction du déplacement en tête

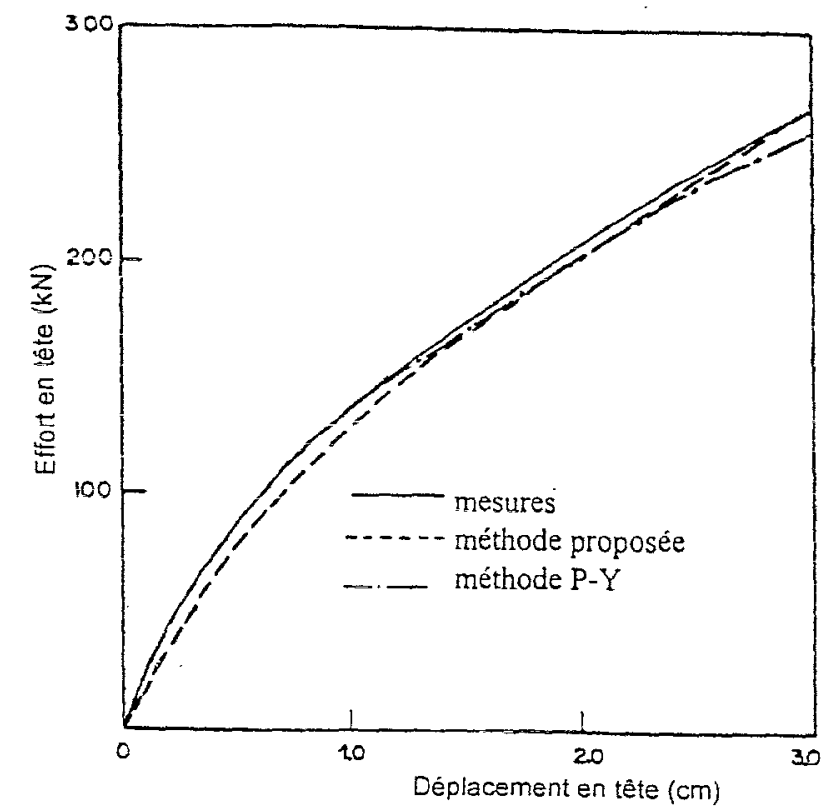

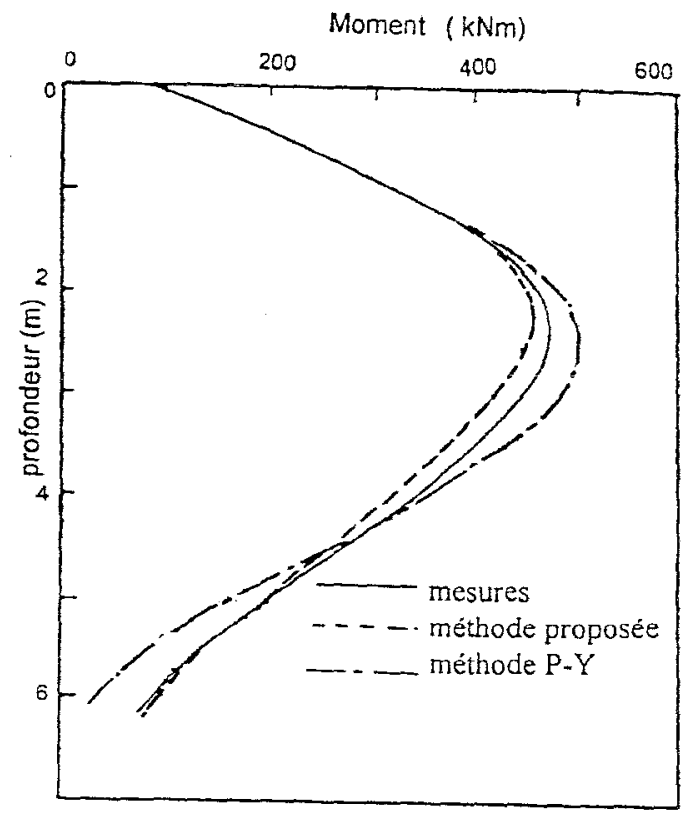

Figure 4 : Moment fléchissant en fonction de la profondeur

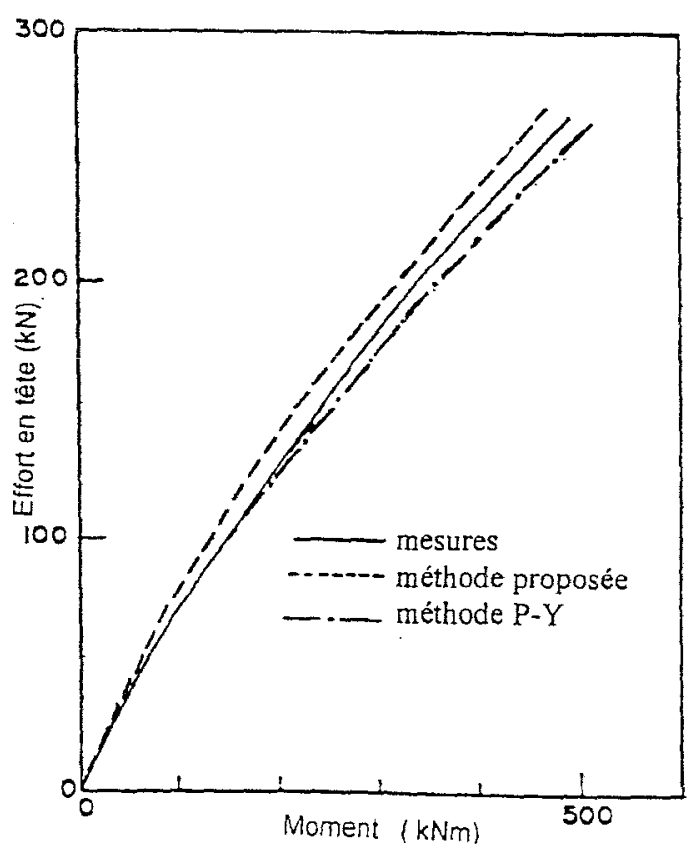

Figure 5 : Moment maximal dans le pieu en fonction de la force en tête 
Figure 6

Force en tête en fonction du déplacement en tête

Figure 7

Force en tête en fonction de la rotation en tête
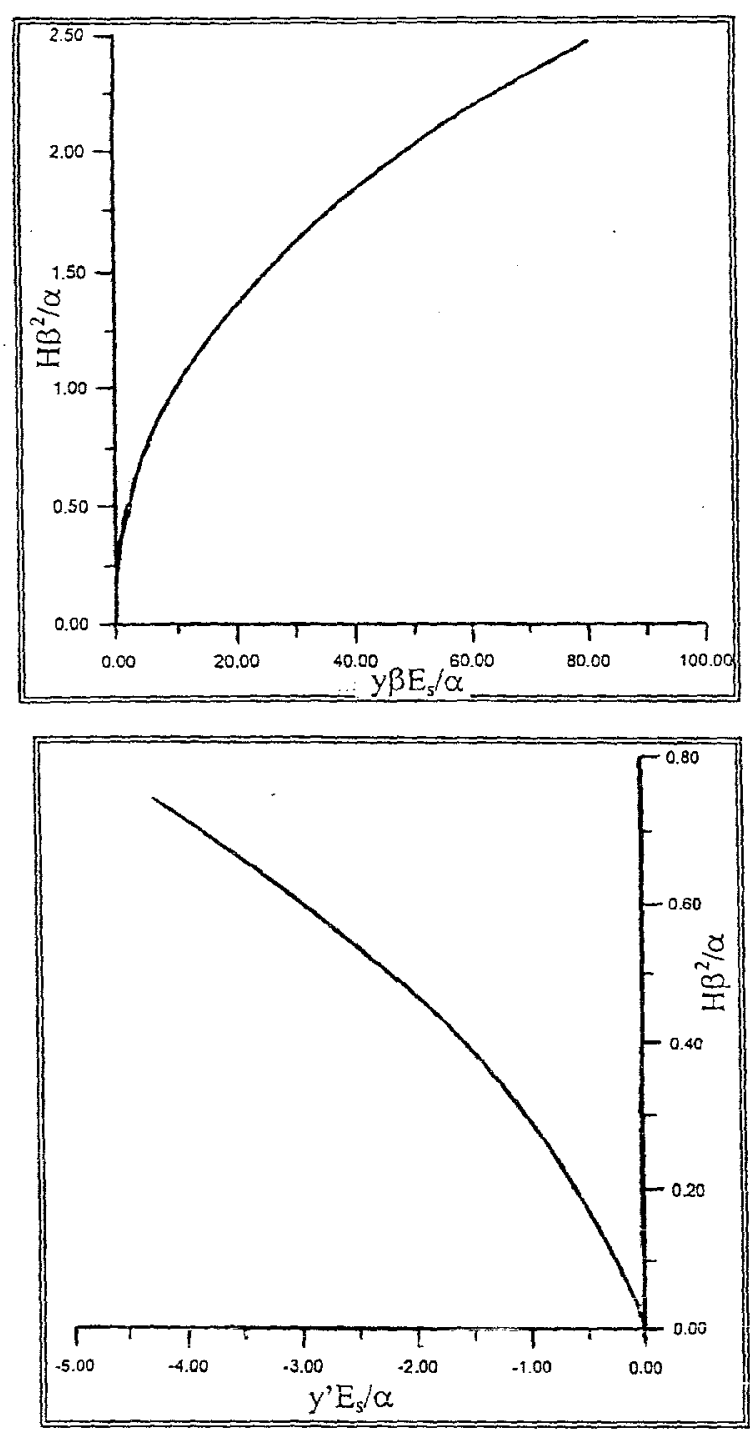

Figure 8

Moment maximal dans le pieu en fonction de la force en tête

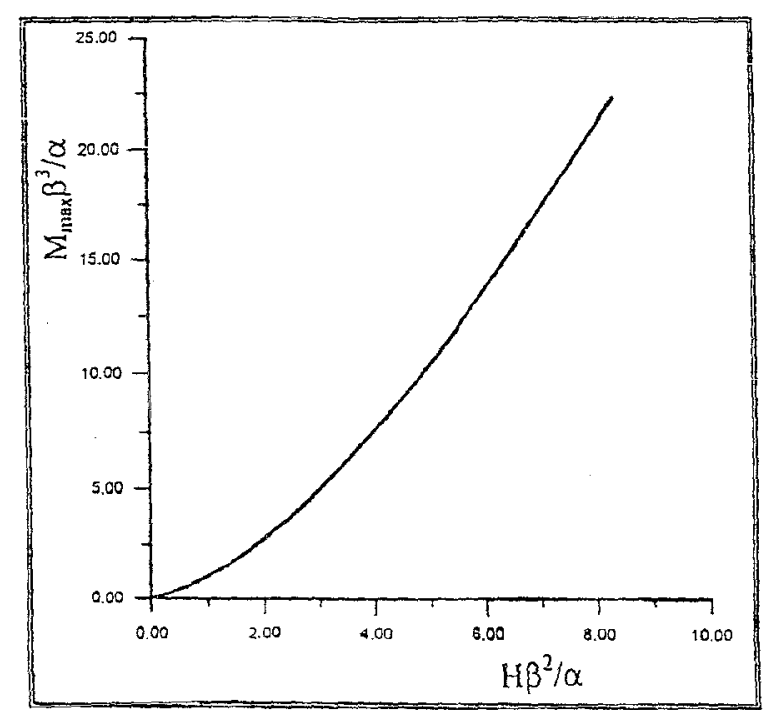




\section{ABAQUES DE RESULTATS}

Le dimensionnement de pieux chargés latéralement nécessite, outre la détermination de la relation effort-déplacement en tête, la connaissance de la distribution des efforts internes $\mathrm{V}(\mathrm{x})$ et $\mathrm{M}(\mathrm{x})$ et surtout la valeur maximale de ce dernier.

En utilisant l'approche proposée, on a montré qu'il est possible de définir respectivement, un déplacement adimensionnel en tête noté y $\beta E s / \alpha$, un moment maximal adimensionnel noté $M_{\max } \beta^{3} / \alpha$, une rotation adimensionnel en tête notée $y^{\prime} E s / \alpha$ qui peuvent s'exprimer en fonction de l'effort adimensionnel en tête, noté $\mathrm{H}^{2} / \alpha$ ou $\mathrm{M} \beta^{3} / \alpha$ selon le chargement, comme le montrent les figures 5,6 et 7 pouir un pieu libre en tête et soumis à un effort horizontal.

Une série d'abaques similaires, donnant directement le déplacement et la rotation en tête ainsi que le moment maximal de flexion dans le pieu, a été obtenue pour différents cas de sol, de chargement et de conditions aux limites.

\section{Conclusion}

Nous avons établi un modèle théorique simple pour l'étude de pieux chargés latéralement, sachant que ce modèle s'applique très bien aux ouvrages de défense. Les solutions analytiques obtenues par ce modèle sont d'un usage aisé et peuvent être intégrées dans un processus de dimensionnement de tels ouvrages. La confrontation avec des résultats d'essais expérimentaux à confirmé la bonne précision de ces solutions.

Le modèle permet de mettre en évidence l'influence de chacun des paramètres de sol et de pieu sur les résultats.

En utilisant ce modèle nous avons développé des abaques donnant directement les relations effort-déplacement en tête, effort-rotation en tête et effort-moment maximal dans le pieu.

Enfin, ce modèle a été utilisé pour le calcul d'un groupe de deux pieux et les résultats sont très encourageants.

\section{Bibliographie}

BARBER E.S (1953), Discussion on load tests on fixed and free-head piles, Symposium on lateral load test on piles, ASTM special technical publication, $N^{\circ} 154,97 \mathrm{p}$.

DAVISSON H.L. (1970), Lateral load capcity of piles, Highway research road, No 333, pp. 104-112.

OTEO C., VALERIO J. (1981), A simplified analysis of pile with lateral loads, X I.C.S.M.F.E, STOCKHOLM 1981, Vol.2, pp. 795-798.

COX W. R., REESE L. C., and GRUBBS. B. R. 1974. Field testing of laterally loaded piles in sand. Proceedings of the Sixth offshore Technology Conference, Houston, TX, Vol. II, pp. 459-472.

RAJANI B. and MORGENSTERN N. Pipelines and laterally loaded piles in elastoplastic medium. J. Geotech. Engrg., ASCE, Vol. 119, No. 9, pp. 1431-1447. 